\title{
The relationship between functional movement analysis and lower- body injury rates in adolescent female football players
}

\author{
D C Janse van Rensburg, MD; A Jansen van Rensburg, MSc; P C Zondi, MB ChB; S Hendricks, BA (Hons) Sport Science; \\ C C Grant, PhD; L Fletcher, PhD \\ Section Sports Medicine, University of Pretoria, Pretoria, South Africa
}

Corresponding author: D C Janse van Rensburg (christa.jansevanrensburg@up.ac.za)

\begin{abstract}
Objective. To determine whether a relationship exists between the functional movement analysis (FMA) score and lower-body injury rates in high-performance adolescent female football players.

Method. Observations included a baseline FMA score and medical injury reports. Data were collected from 24 players' injury and illness records over a 38-week training period. All football injuries requiring medical attention (including stiffness, strains, contusions and sprains) and/or the removal from a session, leading to training restriction, were included in the study. Off-season weeks were excluded. Pearson's product-moment correlation coefficient was calculated to assess the strength of the linear relationship between the FMA score and the number of medical visits, and between the number of medical visits and the number of training-restriction days.

Results. There was no evidence of a relationship between the FMA score and injury risk in teenage female football players $(r=0.016$; $p=0.940$ ). A strong indication of a cyclical season in the training schedule was noticed over the 38 -week study period. A substantive negative correlation $(r=-0.911 ; p=0.032)$ was seen in the number of medical visits compared with the training-restriction days. Injuries during two peak periods could have resulted from overuse, increased training load, stress and overtraining.

Conclusion. It could not be shown that a high FMA score was associated with a lower risk of injury. The ultimate goal is thus to reduce recurrent injury in players with a high FMA count. The regular medical visits observed suggest that player condition is maintained by means of reducing injury and managing training-restriction days. Our findings are in accordance with previous studies in terms of the lower limb being the most frequent region of injury, specifically the knee. This study supports previous suggestions that it is essential to develop a prevention strategy to measure trauma and recovery.
\end{abstract}

S Afr J SM 2013;25(4):109-113. DOI:10.7196/SAJSM.477

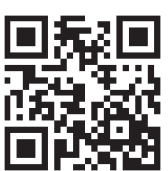

Football, one of the most popular team sports worldwide according to the Fédération Internationale de Footbal Association (FIFA) survey of 2006, is increasing in popularity, especially among young female players. ${ }^{[1]}$ This high participation rate also associates football with a high injury risk, especially in teenage players. Numerous studies have found female players to be more prone to injury than their male counterparts. ${ }^{[2]}$ This is especially true for youth players in competition, as they show a higher injury rate per match than their seniors. ${ }^{[3]}$

Although there have been epidemiological injury studies on female football in recent years, most of these focused on elite female players. ${ }^{[4]}$ There are limited publications available on epidemiological injury records in adolescent female football players. In a 2001 - 2009 study by Waldén et al. ${ }^{[5]}$ female football players showed a two-fold increase in injury incidents compared with their male counterparts in the same age group. Emery ${ }^{[6]}$ and Söderman et al. ${ }^{[7]}$ performed two other studies on injury rates in female adolescent players, focusing on the 12 - 18- and 14 - 19-year age groups, respectively. However, these studies looked only at injury prevalence over $3^{[6]}$ and 7 months, ${ }^{[7]}$ respectively, which may not be sufficient to attain reliable injury incidents, taking all factors of a long season into account. Many of these injuries may have long-term consequences that include an extended phase of rehabilitation and inability to perform sports, as well as the possibility of incomplete recovery that could cause lasting disability for the injured player. Understanding and identifying injury risk factors are essential to developing and improving methods to prevent injuries. ${ }^{[8]}$

Compensatory and incorrect movement tactics are often used by players in an effort to achieve higher performance. These improper actions may emphasise poor body movement patterns during play, resulting in injury. In 2003, the FIFA Medical and Research Centre (F-MARC) $)^{[9]}$ developed a structured training programme, 'The 11, aimed at amateur players aged 13 - 17 years and focused on core stability, lower-extremity strength, neuromuscular control and agility. The objective of this programme was to prevent or lessen injury and thereby possibly enhance performance. A review of the original programme confirmed the potential to decrease injuries, but compelling evidence was not obtained due to poor compliance. Revisions to 'The 11' in 2008, and later in 2010, improved the efficacy of the intervention, with research showing a $30 \%$ reduction in the risk for minor injuries, and as much as a $50 \%$ reduction in the risk for severe injuries such as anterior cruciate ligament (ACL) sprains. ${ }^{[10]}$ As with standard warm-up exercises, this programme should be performed at least twice a week at the start of each training session. Another warm-up prevention programme that has been shown to reduce injuries significantly, specifically non- 
contact ACL injuries, is the Prevent Injury, Enhance Performance (PEP) programme developed by the Santa Monica Orthopaedic and Sports Medicine Research Foundation. This programme has been shown to reduce ACL injuries by up to $60-89 \%$. However, $6-8$ weeks of consistency is required for these changes to be effective. ${ }^{[1]}$

Other intervention programmes have also been shown to reduce injury risks in athletes, if implemented appropriately. The Functional Movement Screen (FMS) is a validated and reliable screening tool consisting of seven different exercises that highlight any functional or biomechanical limitations during specific movement sequences. ${ }^{[8]}$ The athlete is scored from 0 to 3 depending on how well the movement is executed, with 3 being the best. An athlete with a functional limitation or weakness will have a lower total FMS score and is at increased risk of injury when participating in sport. The FMS was designed by Cook $e t$ al. ${ }^{[12]}$ to assess the balance of mobility and stability required to perform essential movements. The movements require specific neuromuscular co-ordination in a range of occupational and physical tasks. Use of the FMS score as a baseline for correcting movement in a rehabilitative setting could remove the primary injury risk factor in predicting performance durability or subsequent injuries. ${ }^{[13]}$ These scores could be used during pre-season or baseline sports physicals to identify athletes with pain or movement limitations before the athletic season commences. Researched factors for injuries include poor mobility, stability, core strength and asymmetries. ${ }^{[8]}$

The functional movement analysis (FMA) programme is based on the FMS, ${ }^{[12]}$ and is designed to evaluate the functional movement ability of an athlete off the field. Additional movements are included for further assessment of posture and pelvic stability. To differentiate from the FMS, the assessment scoring system has also been adapted from 1 to 4 to allow a better reflection of the different competencies, and to monitor improvements of functional movement more effectively.

A training team of 24 adolescent female football players was the target group in this study. The group represented a high-performance squad recruited by national selectors at various tournaments throughout South Africa. The players were selected based on football skill and athletic potential. They were housed at a high-performance unit, where they received coaching and conditioning by national trainers and had unlimited access to sports science and medical interventions.

The objective of this study was to examine the correlation between FMA score and lower-body injury rates in adolescent female football players aged 13 - 18 years over a 38-week training period.

\section{Methods}

\section{Population}

This was a descriptive pilot study based on the results of the highperformance programme of the 2012 SAFA/LOTTO Basetsana Football Academy at the University of Pretoria in South Africa. Twenty-four adolescent female football players participated in the investigation. The players, aged 13 - 18 years, were assigned over a 38-week training period (January to September 2012). This period coincided with the athletes' respective training and tournament season. It excluded the off-season period of October - December 2012.

\section{Injuries and illnesses}

Injury and illness surveillance included assessment by doctors and physiotherapists. Players had free access to the medical staff at all times. Attention was given to all football injuries requiring medical attention (including stiffness, strains, contusions and sprains) and/or the removal from a session, leading to training restriction.

\section{Procedures}

At the start of the study, height, weight and body mass index (BMI) were determined. Observations included a baseline FMA score and medical injury reports. Participants qualified for inclusion in the study if they participated in regular physical activity at a competitive level. Participants were excluded if they used any orthotic device or joint aid (e.g. knee brace), or reported any recent ( $<6$ weeks) musculoskeletal or head injury likely to have an impact on fitness performance on the FMA. Athletes received a zero score if pain was related to any part of the tests.

The FMA analysis is an adaptive version of the FMS as described by Cook et al., ${ }^{[12]}$ and entails ten movement assessments: posture, flexibility, shoulder mobility, one-legged squat, one-legged jump, overhead jump, lunge, rotational stability, trunk stability and bridging. The scoring system on the FMA assessment ranges from 1 to 4 , with 4 being the best possible score. The best total score that can be achieved on the FMA is 40 .

Intra-tester reliability has been shown to be most reliable when testing is performed by one person with a minimum of 6 months of experience in addition to clinical experience. ${ }^{[14]}$ Therefore, in this study, only one tester was used, with 5 - 6 years of experience in the FMS testing procedure.

\section{Statistical analysis}

The Pearson product-moment correlation coefficient, $r$ - a measure of the linear association between two variables - was used to quantify the relationship between FMA and the total number of medical visits, as well as the relationship between the total number of medical visits and training-restriction days. The level of significance was set at the conventional $p \leq 0.05$.

\section{Results}

During the 38-week study period, 24 players were evaluated (Table 1). The overall weight, height, age and BMI of the participants compared well with those of player groups used in similar reported studies. The FMA score rated average according to the FMA score card. During the 38 -week study period, 15 of the 24 players reported a total of 38 medical visits.

A high number of medical visits was not only observed in players with a low FMA score, but across a wide range of FMA scores (Fig. 1). Pearson's correlational analysis confirmed a non-significant relationship $(r=0.016 ; p=0.940)$ between FMA and the number of medical visits. Two distinctive peaks were evident in the number of medical visits during March and August: 13 and 9, respectively (Fig. 2). A slight increase and a definite drop in medical visits was evident in the months preceding and following the peak periods.

Fig. 3 depicts that the documented training-restriction days and the sum of medical visits peaked simultaneously during the months of March and August. The elevated training-restriction peak in March was evidently preceded by a slight increase in February and a gradual decline in April. Pearson's correlational analysis confirmed a significant relationship ( $p=0.032 ; r=-0.911$ ) between the reported number of 
Table 1. Baseline physical characteristics of female participants $(13$ - 18 years $)(N=24)$

Age (years), mean $( \pm \mathrm{SD})$

$15.7( \pm 1.3)$

Body weight $(\mathrm{kg})$, mean $( \pm \mathrm{SD})$

$54.9( \pm 9.04)$

Height $(\mathrm{cm})$, mean $( \pm \mathrm{SD})$

BMI $\left(\mathrm{kg} / \mathrm{m}^{2}\right)$, mean $( \pm \mathrm{SD})$

$164.0( \pm 5.95)$

FMA score, mean $( \pm S D)$

$20.3( \pm 2.61)$

Total medical visits, $n$

Players who reported medical visits, $n$

Total training restriction days, $n$

Study period (weeks)

29

38

FMA = functional movement analysis.

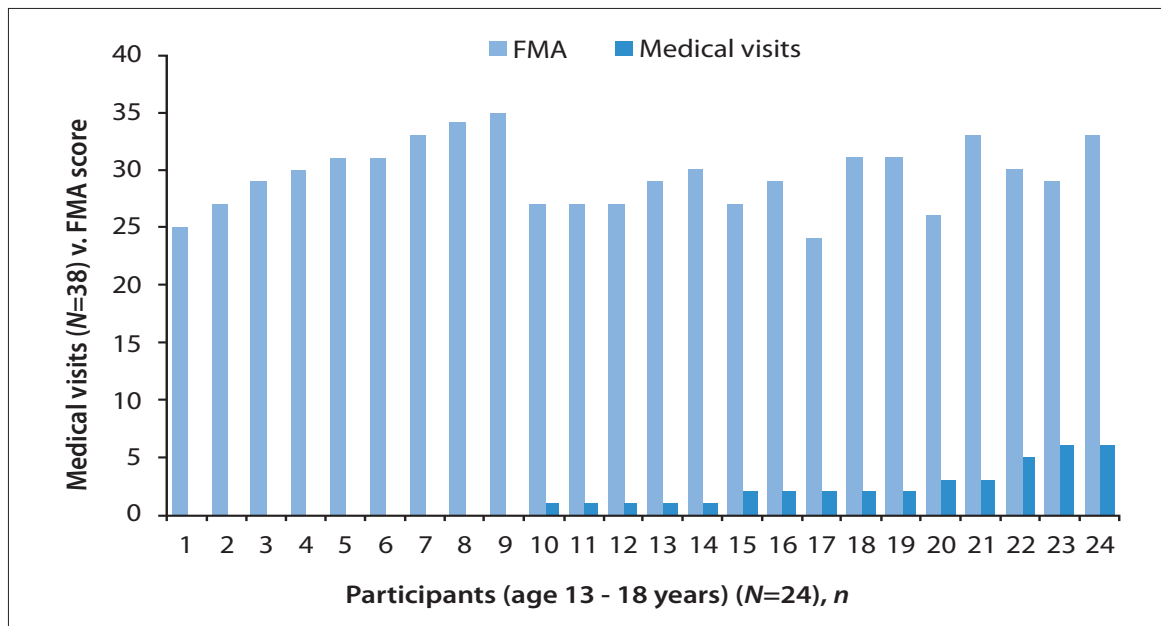

Fig. 1. Number of medical visits in the 38-week study period v. total FMA score.

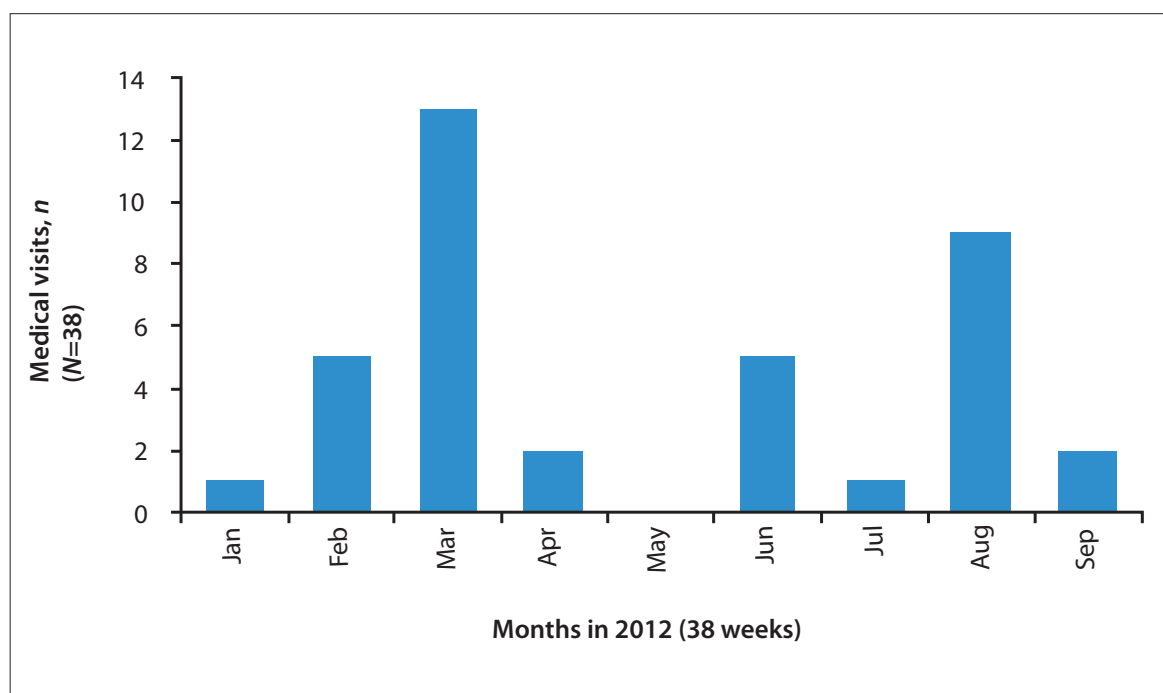

Fig. 2. Number of medical visits over the 38-week study period.

medical visits and the number of trainingrestriction days.

Training restriction was placed mainly on injuries in the knee, foot and ankle regions (Fig. 4). However, during the 38-week period, the knee followed by the lower leg, the foot and ankle regions, where training restrictions were recorded.

The mechanism of injury, in particular 'overuse' and 'forced extension', peaked during the months of March, and 'overuse' peaked again in August (Fig. 5). Overuse was graded as a gradual-onset injury, while forced extension, flexion and rotation, and blunt injury were categorised as acute-onset injuries.

Muscle stiffness and strains accounted for 16 injuries (28\% each) and were the most frequent occurring specific injury types identified. Other injury types were sprains $(17 \%)$ and contusions (10\%).

\section{Discussion}

There was no significant correlation between FMA score and injury risk in the players. However, a strong negative correlation $(r=-0.911 ; p=0.032)$ was found between the number of medical visits and the trainingrestriction days. A strong indication of a cyclical season in the training schedule was noticed during the 38-week period. Lowerbody injuries in these two peak periods could have resulted from overuse, increased training load, stress and overtraining.

In previous studies, a lower FMS score had a predictive ability and was a predisposing factor for injury in male participants in active military service, ${ }^{[15]}$ as well as in male athletes in American Football. ${ }^{[8]}$ The same is true for female athletes, although not necessarily female football athletes, with very few studies in this regard. ${ }^{[13]}$ Further studies, such as this one, are warranted to determine FMS scores and injury prevalence among young female athletes. Previous research has confirmed that the screening of movement patterns can readily identify functional limitations and asymmetries, and can be used to lower injuries in high performers. ${ }^{[8]}$ In the current study, the FMA as a score card was assessed relative to the number of recorded medical visits among the football players. However, the correlational analysis did not substantiate that a high FMA score would directly lead to fewer medical injuries.

The number of medical visits and trainingrestriction days peaked during the months of March and August. This could indicate a cyclical season in the training schedule of the players, during which injuries could have resulted from overuse, increased training load, stress and overtraining. The training- 


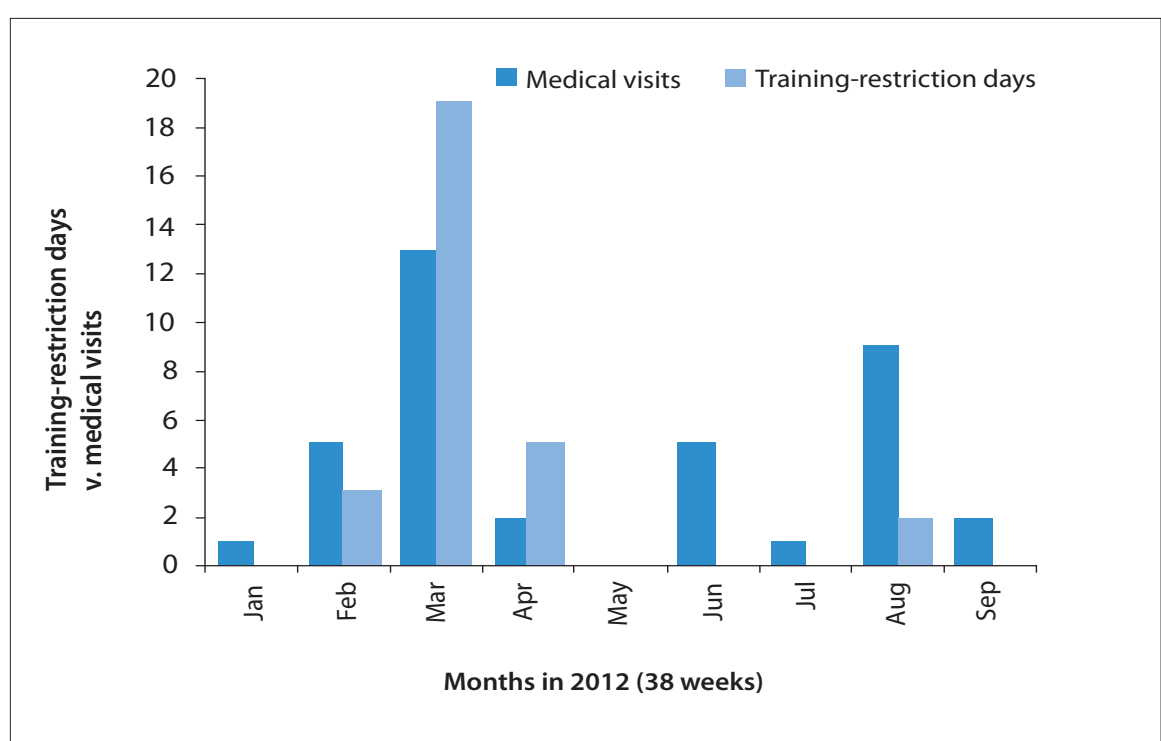

Fig. 3. Number of medical visits v. training-restriction days over the 38-week study period.

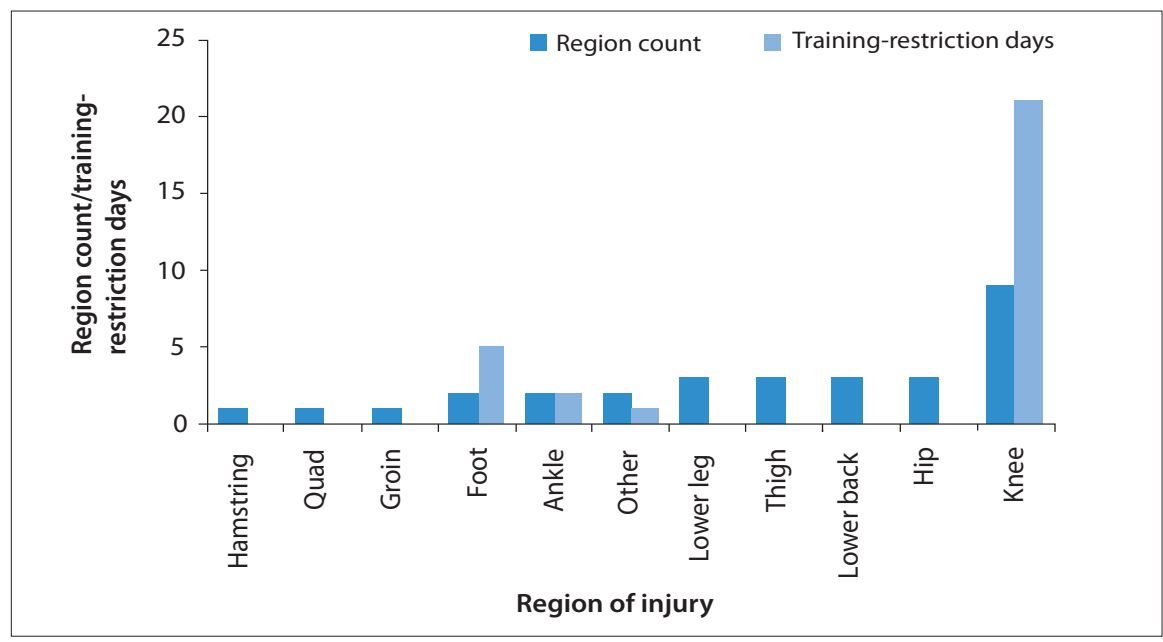

Fig. 4. Region of injury v. training-restriction days in the 38-week study period.

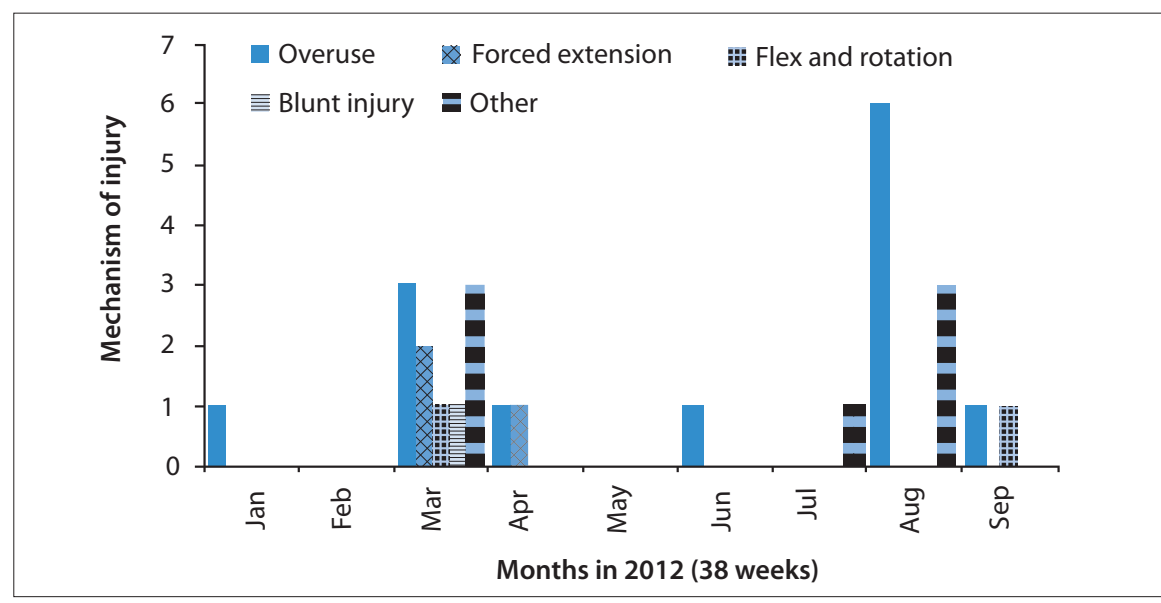

Fig. 5. Mechanism of injury over the 38-week study period.

restriction peaks could also have been due to the fact that many of the players represented their respective U17 and U20 national teams

during the same year. It has been shown that it is during competition where most incidences of injuries in female footballers occur. ${ }^{[3]}$ The specific time-frame of this research coincided with the respective training and tournament seasons of the players, stretching over a 38week period. Baseline training and fitness occurred during January and February. More sport-specific skills training with increased load (i.e. quality and quantity specific) commenced in March, with mid-week and weekend matches in August. The study period excluded the off-season segment of 3 months. The fact that a strong, negative correlation was found between the number of medical visits and training-restriction days further indicates that regular medical visits may maintain player condition in reducing injury and managing total restriction days.

Injuries in football players have significant implications, not only in relation to future participation in physical activity, but also in future morbidity and mortality related to physical inactivity. Therefore, the reduction of injuries is critical. Injury prevention or a protective intervention training programme will help to lower football-related injuries. According to Emery et al., ${ }^{[16]}$ the risk of injury in youth indoor football players can be reduced by more than one-third with a football-specific neuromuscular training programme. Also with football being a team sport and players required to be at a high level of performance for the season, variation in training should be considered. Periodisation or fluctuation of high and low training loads (including changes in volume, intensity and strength training) within a small time-period, as well as balanced periods of rest and recovery, have proven to benefit performance and reduce the risk of injury. ${ }^{[17]}$

In this study, the knee was the most injured region followed by the lower leg, thigh, lower back and hip. The lower leg, thigh, lower back and hip injuries were, however, less serious, since training restrictions were placed mainly on injuries of the knee, foot and ankle. The knee, foot and ankle regions can therefore be considered to be highly vulnerable areas of the football player's body, which may also lead to lengthy exercise-restriction periods in the event of injury. This is consistent with other studies listing the most common injury locations in female football players as the knee, ankle and thigh. ${ }^{[18]}$ Improved preparation and training, and a structured workout schedule with a specific area of focus, will ensure reduced training restraints of athletes. ${ }^{[19]}$ 
The high rate of injury seen in a recurrent or increased pattern over specific months suggests the need for the implementation of a specific injury-prevention training approach. Soligard et al..$^{[10]}$ in 2008 indicated that players, in particular young female football players, could only benefit from proper biomechanical technique and improved awareness exercises to lower the risk of injury. Lerch et al. ${ }^{[20]}$ is in agreement with this in his literature review demonstrating the effectiveness of injuryprevention programmes in female youth football players. Due to the results of the mechanism of injury reported in this study showing overuse injuries as a peak, along with the suggestion of the cyclical seasoning in the training schedule of the players, it would be beneficial for the trainers to utilise different methods of quantifying training load - so as to provide a more quantitative measure of internal load on each player, to avoid overuse injuries and over-reaching. ${ }^{[21]}$ While this will not affect performance, ${ }^{[22]}$ it will allow for reliable internal load to be recorded and specific training prescription to be given accordingly, ${ }^{[23]}$ which, in the long term, will assist in injury prevention and consequently player longevity.

Musculoskeletal injuries accounted for the majority of injuries in training. The most frequent occurring specific injury types were stiffness and strains. Although sprains and contusions occurred less frequently, these specific injury types can also be classified as acute-onset injuries. Thus, it seems that acute-onset injuries are a bigger concern than gradual-onset injuries. This varies, with previous research showing that young female footballers tended to sustain fewer strain injuries and an increased number of ligament injuries than their male counterparts of equivalent age. ${ }^{[24]}$

\section{Conclusion}

It could not be shown that a high FMA score was associated with a lower risk of injury. The ultimate goal will thus be to reduce recurrent injury in players with a high FMA count. Our results agree with previous studies in terms of the most frequent region of injury being the lower limb, specifically the knee. Our study thus supports previous suggestions that it is essential to develop a prevention strategy to measure trauma and recovery.

Trainers, coaches and medical staff may well consider monitoring the female football-training programme, the training load, the duration of training as well as the matches and the psychosocial recovery (monitoring the stress and strain) of each player. Due to the large variation between players, the individual variances over time are most substantial in relation to injury prevention. Trainers need, therefore, to gain information on an individual basis to identify when a player has an increased risk of injury. If necessary, the training schedule can be adapted or interventions used in which players are educated to manage injury in a better manner.

Areas of future study include quantitative recording of the training programme to correlate high peak injury occurrences with high peak training cycles. Recording and quantifying the internal load and recovery of players will allow future researchers to deduce more precise risk factors and trends for injury prevalence. While this study looked at the trends during a 38-week training period, it may be more reliable for future researchers to track similar trends over multiple seasons, allowing an intricate analysis into the cyclical training, season by season. Increased intra-tester reliability for FMS testing will strengthen future studies.
Acknowledgements. Amy Bathgate is acknowledged for assistance with data collection.

\section{References}

1. FIFA. FIFA big count 2006: 270 million people active in football. http://www.fifa.com/ aboutfifa/media/newsid=529882.html (accessed 14 January 2013).

2. Emery CA, Meeuwisse WH, Hartmann S. Evaluation of risk factors for injury in adolescent soccer: Implementation and validation of an injury surveillance system. Am J Sport Med 2005;33:1882-1891.

3. Junge A, Dvorak J. Injuries in female football players in top-level international tournaments. Br J Sports Med 2007;41(Suppl 1):i3-i7.

4. Faude O, Junge A, Kindermann W, Dvorak J. Injuries in female soccer players: A prospective study in the German national league. Am J Sports Med 2005;33(11):16941700 .

5. Waldén M, Hägglund M, Magnusson H, Ekstrand J. Anterior cruciate ligament injury in elite football: A prospective three-cohort study. Knee Surg Sports Traumatol Arthrosc 2011;19(1):11-19. [http://dx.doi.org/10.1007/s00167-010-1170-9]

6. Emery CA. Injury prevention and future research. Med Sport Sci 2005;49:170-191. [http://dx.doi.org/10.1159/000084289]

7. Söderman K, Adolphson J, Lorentzon R, Alfredson H. Injuries in adolescent female players in European football: A prospective study over one outdoor soccer season. Scand J Med Sci Sports 2001;11(5):299-304

8. Kiesel K, Plisky PJ, Voight M. Can serious injury in professional football be predicted by a preseason Functional Movement Screen? N Am J Sports Phys Ther 2007;2(3):147158.

9. F-MARC. http://www.fifa.com/aboutfifa/footballdevelopment/medical/aboutus/ fmarc/index.html and http://www.fifa.com/mm/document/footballdevelopment/ medical/01/47/88/06/f-marcfootballforhealth.pdf (accessed 14 January 2013).

10. Soligard T, Nilstad A, Steffen K, et al. Compliance with a comprehensive warm-up programme to prevent injuries in youth football. Br J Sports Med 2010;44(11):787793. [http://dx.doi.org/10.1136/bjsm.2009.070672]

11. Mandelbaum BR, Silvers HJ, Watanabe DS, et al. Effectiveness of a neuromuscular and proprioceptive training program in preventing anterior cruciate ligament injuries in female athletes: 2-year follow-up. Am J Sports Med 2005;33(7):1003-1010. [http:// dx.doi.org/10.1177/0363546504272261]

12. Cook G, Burton L, Hoogenboom B. Pre-participation screening: The use of fundamental movements as an assessment of function - Part 1. N Am J Sports Phys Ther 2006;1(2):62-72.

13. Chorba RS, Chorba DJ, Bouillon LE, Overmyer CA, Landis JA. Use of a functional movement screening tool to determine injury risk in female collegiate athletes. N Am J Sports Phys Ther 2010;5(2):47-54.

14. Gribble PA, Brigle J, Pietrosimone BG, Pfile KR, Webster KA. Intrarater reliability of the functional movement screen. J Strength Cond Res 2013;27(4):978-981. [http:// dx.doi.org/10.1519/JSC.0b013e31825c32a8 ]

15. Teyhen DS, Shaffer SW, Lorenson CL, et al. The Functional Movement Screen: A reliability study. J Orthop Sports Phys Ther 2012;42(6):530-540.

16. Emery CA, Meeuwisse WH. The effectiveness of a neuromuscular prevention strategy to reduce injuries in youth soccer: A cluster-randomised controlled trial. $\mathrm{Br} J$ Sports Med 2010;44:555-562. [http://dx.doi.org/10.1136/bjsm.2010.074377]

17. Lambert MI. Periodisation and Monitoring of Overtraining in Rugby Players. http:// www.sarugby.co.za/boksmart/pdf/BokSmart\%20-\%20Periodisation\%20and\%20 monitoring\%20of\%20overtraining\%20in\%20rugby\%20players.pdf (accessed 14 January 2014).

18. Giza E, Mithofer K, Farrell L, Zarins B, Gill T. Injuries in women’s professional soccer Br J Sports Med 2005;39(4):212-216. [http://dx.doi.org/10.1136/bjsm.2004.011973]

19. Le Gall F, Carling C, Reilly T. Injuries in young elite female soccer players: An 8-season prospective study. Am J Sports Med 2008;36(2):276-284.

20. Lerch C, Cordes M, Baumeister J. Effectiveness of injury prevention programs in female youth soccer: A systematic review. Br J Sports Med 2011;45:359-359. [http:// dx.doi.org/10.1136/bjsm.2011.084038.140]

21. Alexiou $\mathrm{H}$, Coutts AJ. A comparison of methods used for quantifying internal training load in women soccer players. Int J Sports Physiol Perform 2008;3(3):320-330.

22. Brink MS, Nederhof E, Visscher C, Schmikli SL, Lemmink KA. Monitoring load, recovery, and performance in young elite soccer players. J Strength Cond Res 2010;24(3):597-603. [http://dx.doi.org/10.1519/JSC.0b013e3181c4d38b]

23. Impellizzeri FM, Rampinini E, Coutts AJ, Sassi A, Marcora SM. Use of RPE-based training load in soccer. Med Sci Sports Exerc 2004;36(6):1042-1047.

24. Junge A, Cheung K, Edwards T, Dvorak J. Injuries in youth amateur soccer and rugby players - comparison of incidence and characteristics. Br J Sports Med 2004;38(2):168 172. [http://dx.doi.org/10.1136/bjsm.2002.003020] 